\title{
Norois
}

Environnement, aménagement, société

$235 \mid 2015$

La directive cadre sur l'eau à l'échéance 2015. Les

façades océaniques

\section{L'application de la DCE dans les départements et régions d'outre-mer : la mise en lumière des discordances entre le concevable et le possible à la Martinique}

Application of the EU Water Framework Directive in the French Overseas

Departments: highlighting discrepancies between the conceivable and the possible in Martinique, French West Indies

Guillaume Lalubie, Jean-Raphael Gros-Désormeaux et Thierry Nicolas

\section{CpenEdition}

\section{Journals}

Édition électronique

URL : http://journals.openedition.org/norois/5614

DOI : $10.4000 /$ norois. 5614

ISBN : 978-2-7535-4775-9

ISSN : $1760-8546$

Éditeur

Presses universitaires de Rennes

Édition imprimée

Date de publication : 15 novembre 2015

Pagination : 51-66

ISBN : 978-2-7535-4771-1

ISSN : 0029-182X

Référence électronique

Guillaume Lalubie, Jean-Raphael Gros-Désormeaux et Thierry Nicolas, «L'application de la DCE dans les départements et régions d'outre-mer : la mise en lumière des discordances entre le concevable et le possible à la Martinique », Norois [En ligne], 235 | 2015, mis en ligne le 15 novembre 2017, consulté le 20 avril 2019. URL : http://journals.openedition.org/norois/5614 ; DOI : 10.4000/norois.5614 

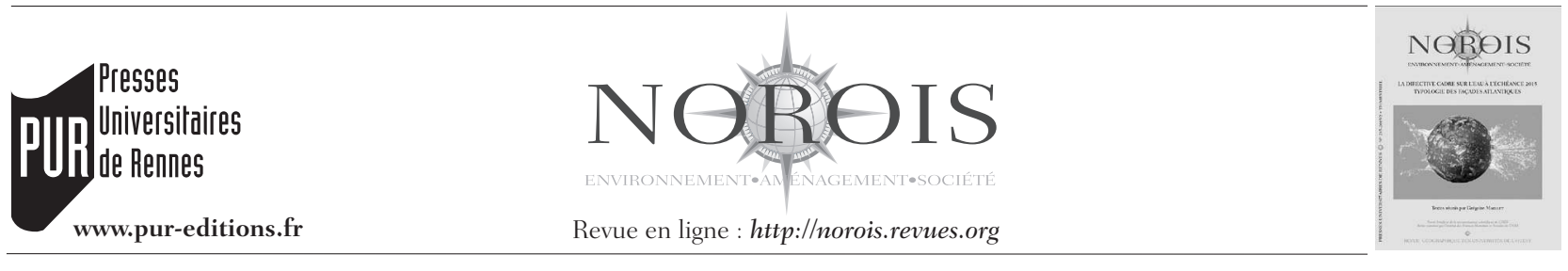

\title{
L’application de la DCE dans les départements et régions d'outre-mer : la mise en lumière des discordances entre le concevable et le possible à la Martinique
}

\author{
Application of the EU Water Framework Directive in the French Overseas Departments: \\ Highlighting Discrepancies between the Conceivable and the Possible in Martinique, \\ French West Indies
}

\section{Guillaume Lalubie ${ }^{* a}$, Jean-Raphaël Gros-Désormeaux ${ }^{\mathrm{b}}$ et Thierry Nicolas ${ }^{\mathrm{c}}$}

\author{
*Auteur correspondant \\ a EA 929, AIHP-GEODE - BP 7207, université des Antilles, 97275 Schclcher cedex, France. \\ (g.lalubie@hotmail.fr) \\ b UMR-CNRS 8053, CRPLC - BP 7207, université des Antilles, 97275 Schelcher cedex, France. \\ (jrgrosde@martinique.univ-ag.fr) \\ cCentre IRD de Cayenne-BP 165,97323 Cayenne Cedex, France.(thierrynicolas@wanadoo.fr)
}

\begin{abstract}
Résumé : Dans un contexte de changements environnementaux globaux, la question de la gestion de l'eau est d'autant plus exacerbée dans les départements et régions d'outre-mer français (Drom). Les savoirs incertains relatifs au contexte de changement global sont en contradiction avec le concept de «conditions de référence » mis en œuvre par la directive cadre européenne sur l'eau (DCE). Les contextes, où incertitude et risque s'entremêlent, autorisent l'hypothèse d'une probable discordance entre des injonctions prévues par la DCE et les possibilités de gestion autorisées par les situations locales. L'objectif de cette contribution est d'interroger les situations Dromiennes sur leur capacité à répondre aux injonctions de la DCE. Si l'application de la DCE dans les Drom a effectivement été porteuse d'efforts soutenus dans la concertation territoriale et l'acquisition de connaissances scientifiques, l'insuffisance de données scientifiques historiques ne permet pas de définir des « conditions de référence » selon une conception métastable de l'équilibre des écosystèmes. L'exemple de la Martinique illustre les difficultés que rencontrent les Drom à donner sens au concept de « conditions de référence » difficilement définissable à cause d'incertitudes dans les savoirs technico-scientifiques. Au delà de cette question, l'inertie autour de la mise en œuvre d'actions concrètes est d'autant plus exacerbée dans ces territoires. Les situations conjoncturelles où incertitude des savoirs, risque et principe de précaution s'entremêlent, sont favorable à accroître les durées de mise en œuvre de l'action collective. Les organisations compétentes sur la gestion des milieux aquatiques dans les Drom gagneraient à investir les instruments financiers qui dynamiseraient la préservation et l'amélioration de l'état écologique des systèmes aquatiques, à partir de « conditions de référence » notamment instituées selon les valeurs socio-écologiques des systèmes aquatiques.
\end{abstract}

Abstract: In a context of global environmental change, the issue of water management is aggravated in the French overseas departments. Uncertain knowledge on the context of global change is in contradiction with the concept of "reference conditions" implemented by the European Water Framework Directive (WFD). This context, where uncertainty and risk are intertwined, raises the hypothesis of a possible discrepancy between the obligations set by the WFD and the management options permitted by local conditions. The objective of this paper is to examine the situation in Overseas Departments and their ability to meet the requirements of the WFD. If the implementation of the WFD in the Overseas Departments was followed by major efforts in local consultation and the acquisition of scientific knowledge, the lack of historical scientific data prevents the determination of "reference conditions" as a metastable conception of the ecosystems' balance. The case of Martinique illustrates the challenges faced by Overseas Departments to ascertain the concept of "reference condi- 
tions", which is difficult to define due to uncertainties of the technical and scientific knowledge. Beyond this issue, the inertia around the implementation of concrete actions is exacerbated in those territories. Cyclical conditions, where uncertainty of knowledge, risk and the precautionary principle interlace, are prone to increase the duration of implementation of collective actions. Relevant institutions in the management of aquatic environments in the Overseas Departments would benefit from using financial instruments that could boost the preservation and improvement of the ecological status of aquatic systems from "reference conditions" defined by the socio-ecological values of the aquatic systems.

Mots clés : DCE, politique de l'eau, gestion des ressources en eau, dispositif institutionnel, Départements d'outre-mer.

Keywords: EWFD, water policy, water management, institutional framework, French overseas departments.

\section{INTRODUCTION}

Les départements et régions d'outre-mer français sont des territoires tropicaux répartis entre l'arc insulaire des Petites Antilles pour la Guadeloupe et la Martinique, le plateau amazonien pour la Guyane et l'océan Indien pour Mayotte et la Réunion (figure 1). Dans un contexte de changements environnementaux globaux, la question de la gestion de l'eau est d'autant plus exacerbée sur ces espaces insulaires (Magnan, 2009). Gabrielle Bouleau et Didier Pont (2014) démontrent que les savoirs incertains relatifs au contexte de changement global sont en contradiction avec le concept de «conditions de référence ${ }^{1}$ » mis en œuvre par la directive cadre européenne sur l'eau, la DCE. S'inscrivant dans la tendance actuelle des New Public Management (NPM), ce concept a pour objet de donner sens aux enjeux de préservation et d'amélioration de l'état écologique des systèmes aquatiques, tout en autorisant des usages durables, ainsi que l'approvisionnement en eau pour les populations.

Si l'application de la DCE dans les Drom est porteuse d'avancées notables, les contextes problématiques, où incertitude et risque s'entremêlent au sein de systèmes socio-écologiques (SES) complexes, autorisent l'hypothèse d'une probable discordance entre des injonctions prévues par la DCE et les possibilités de gestion autorisées par les situa-

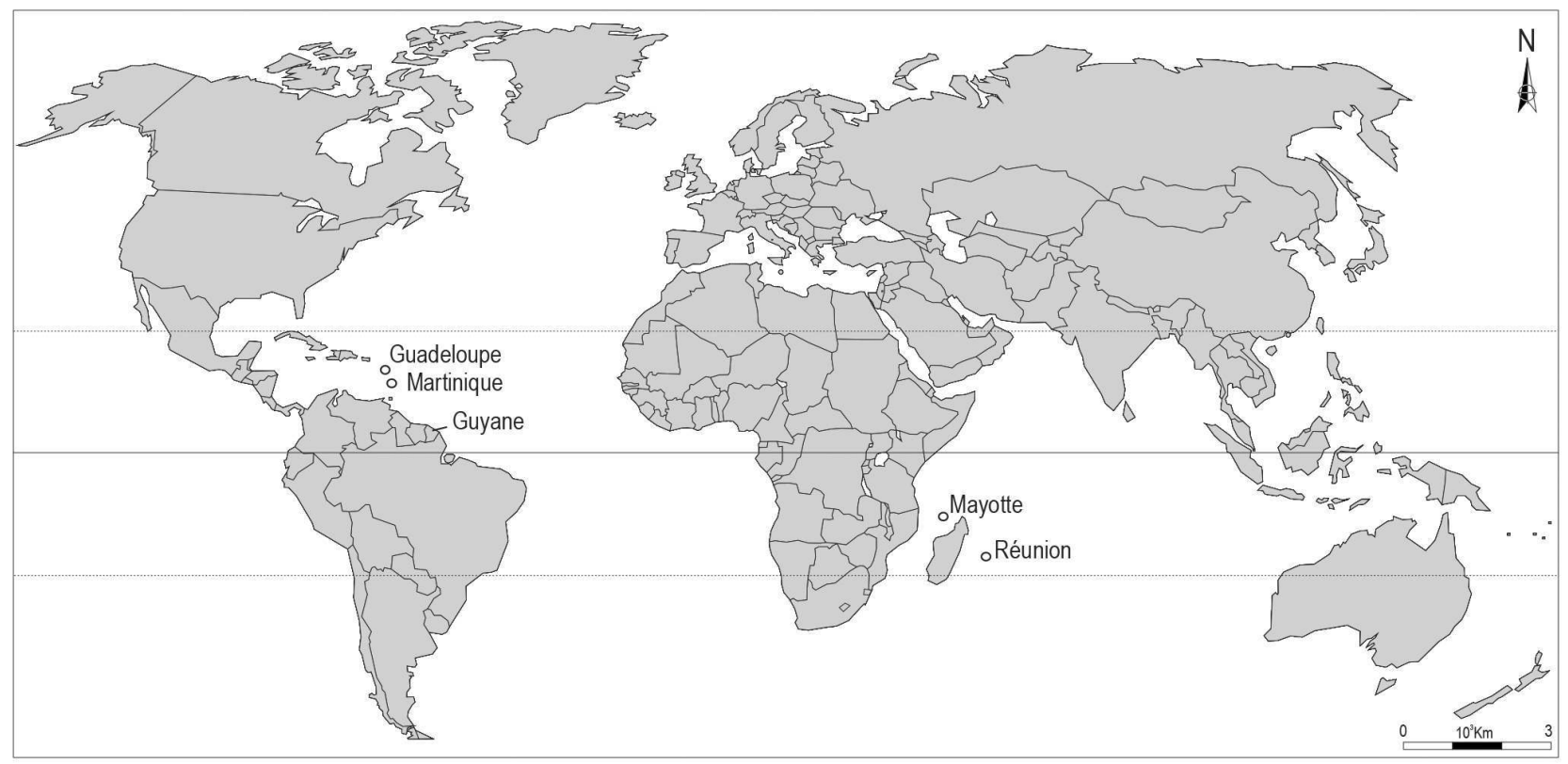

1. Les conditions de référence définissent une situation dans laquelle une eau de surface ne subit pas de pressions anthropiques. Le glossaire de la DCE précise leurs modalités de détermination : les réseaux de référence,

Figure 1 : Localisation des Départements et Régions d'Outre-Mer français

Localisation of the French Overseas Departments 
tions locales. En s'appuyant sur des démarches ethnographiques centrées sur les acteurs, ainsi que sur l'analyse critique des expertises et des schémas directeurs d'aménagement et de gestion des eaux (Sdage), l'objectif de cette contribution est d'interroger les situations dromiennes sur leur capacité à répondre aux injonctions de la DCE, à partir de la critique socio-écologique proposée par Gabrielle Bouleau et Didier Pont sur les « conditions de référence de la directive cadre européenne sur l'eau face à la dynamique des hydrosystèmes et des usages » (Bouleau et Pont, 2014). Après une description de la mise en application de la DCE dans les orientations des Sdage dromiens, la réflexion se propose de mettre l'accent sur les éléments contextuels communs à l'ensemble des Drom qui sont de nature à contrarier des injonctions de la directive, puis d'approfondir l'analyse à partir des difficultés d'applicabilité qui sont propres à la Martinique.

\section{LA MISE EN APPLICATION DE LA DCE DANS LES ORIENTATIONS DU SDAGE : DE LA DIRECTIVE À SA PLANIFICATION LOCALE}

\section{Décryptage de la DCE par l'intermédiaire des Sdage}

L'objectif de la DCE est d'établir un cadre pour la protection des eaux au sein des territoires de l'Union européenne (UE). Ce cadre se décline en huit types d'actions : prévenir, préserver, améliorer, promouvoir, renforcer, assurer et contribuer. Les objectifs de préservation et d'amélioration concernent l'état des eaux dans sa globalité, particulièrement d'un point de vue écologique. En outre, les actions liées à la prévention, à l'amélioration, à la promulgation, au renforcement et à l'assurance sont spécifiquement liées aux pollutions des eaux. Enfin, l'objectif de contribuer à atténuer les effets des inondations et des sécheresses tente d'inciter les pays membres à des initiatives de mitigation des effets prévisionnels des changements climatiques, comme l'accroissement de la fréquence des événements extrêmes.

Malgré leurs hétérogénéités socioculturelles, économiques et géographiques, des aspects communs peuvent se retrouver dans les différents Drom. Dans chaque territoire, en congruence avec l'article 13 de la DCE, un plan de gestion de l'eau à l'échelle du district hydrographique fut établi en 2009, sous la forme d'un Sdage révisé tous les 6 ans. Dans ces territoires ultra-marins, les districts, qualifiés à tort « d'hydrologiques », sont délimités par les frontières du département : ils correspondent donc à une île (Martinique, Mayotte, Réunion), un archipel (Guadeloupe) ou à une partie de continent (Guyane). Prévus nationalement par la loi sur l'eau du 3 janvier 1992, les premiers Sdage, réalisés sous l'égide d'un Comité de bassin dans un esprit de démocratie participative, ont été validés au début des années 2000, exception faite de Mayotte qui n'était pas encore département à cette période. Ces Sdage fixaient les orientations fondamentales d'une gestion globale et équilibrée de la ressource en eau. En cohésion avec les injonctions de la DCE, les Sdage de 2009 à 2015 inclus des objectifs quantifiés de qualité à atteindre, concernant l'hydrologie, l'hydro-géomorphologie, la physico-chimie et la biocénose. Conformément à la directive, ces objectifs de qualité devront être atteints au mieux en 2015, ou encore par dérogation motivée en 2021, mais, en tout état de cause, l'ensemble des masses d'eaux devra impérativement correspondre aux critères de bonne qualité à l'échéance 2027.

Les Sdage ${ }^{2}$ qui couvrent la période de 2009 à 2015 reflètent les politiques de gestion de l'eau dans les territoires ainsi que les orientations formulées en vue d'atteindre les objectifs de la DCE. L'analyse comparative des orientations et des sousorientations fondamentales de ces Sdage permet de constater la récurrence des questions relatives à la lutte contre les pollutions, l'amélioration de l'assainissement collectif et individuel, la gestion quantitative de l'eau pour l'ensemble des usages dans les îles, ainsi que les risques hydro-géomorphologiques. En plus des risques d'inondation et d'érosion (glissement de terrain et lave torrentielle), le risque sanitaire apparaît également comme étant un problème particulièrement prégnant dans les Sdage de Guyane et de Mayotte. La question d'une gouvernance efficiente est également commune à l'ensemble des Sdage dromiens, à l'exception de la Martinique qui n'aborde pas cette problématique. La préservation, la restauration et la conservation des écosystèmes naturels d'eau douce ou salée font

\footnotetext{
2. [http://www.comite-de-bassin-guadeloupe.fr]; [http://www.bassin-guyane.fr]; [http://www.officedeleau.fr]; [http://www.mayotte.pref.gouv.fr]; [http://www. comitedebassin-reunion. fr $]$.
} 
l'objet d'une orientation fondamentale spécifique dans les Sdage de la Guadeloupe, de Mayotte et de la Réunion. Dans les deux autres territoires, les mesures équivalentes sont incluses dans d'autres orientations fondamentales. De même, l'amélioration de la connaissance scientifique, prioritaire dans les Drom, est formulée en orientation fondamentale dans les Sdage de Guyane et de Martinique uniquement. La promotion des pratiques écocitoyennes, à titre individuel ou par branche d'activité, fait l'objet d'une mesure fondamentale pour la Martinique et Mayotte. Enfin, seul le Sdage de la Guadeloupe accorde une attention prioritaire à la problématique des pesticides.

Les injonctions de la DCE dans les Sdage des Drom sont donc traduites par deux principales thématiques : la restauration de la qualité hydrologique, physique, chimique et biologique des eaux; et la gestion intégrée de l'eau pour satisfaire aux multiples besoins naturels et anthropiques, tout en limitant les conflits d'usage. Dès lors, des mesures comme la lutte contre les pollutions, la gestion de la ressource en eau, la gestion des écosystèmes aquatiques, le suivi de la qualité des milieux aquatiques, ainsi que l'amélioration de la connaissance scientifique, répondent parfaitement à la directive.

\section{Les avancées procurées par la DCE}

À partir des années 2000, la DCE a été un catalyseur efficace de mise en place d'actions concrètes de gestion des milieux aquatiques dans les Drom. Les objectifs de bon état quantifiable des masses d'eaux, au mieux en 2015, mais surtout pour 2027, imposent donc un effort constant des gestionnaires. Ces injonctions, planifiées par l'UE, ont contribué à dynamiser la gestion des eaux dans les Drom.

Depuis la préparation du Sdage de 2009, il a été constaté la récurrence de forums hybrides consacrés à la gestion de l'eau dans les Drom. Dans une volonté de gestion intégrée de l'eau, les sphères économiques, associatives et administratives ont adhéré aux démarches de consultation motivées par les Sdage. Ces espaces de dialogue territorial ont été des opportunités pour résoudre les conflits d'usage, par la fabrique institutionnelle de plans de gestion comme les contrats de rivière (contrat de rivière du Galion à la Martinique) et les contrats de baie (contrat de baie de Fort-de-France à la Martinique).
Plus encore, la DCE recommande d'améliorer la connaissance scientifique en vue de comprendre et de suivre les équilibres hydrologiques, morphodynamiques, physico-chimiques et écosystémiques. De ce fait, elle a fortement impulsé la dynamique d'acquisition de connaissances scientifiques de milieux aquatiques, constatée au début du $\mathrm{XxI}^{\mathrm{e}}$ siècle dans les Drom. Ceci a permis le développement ou la création de bureaux d'étude locaux et l'implantation d'antennes de grands bureaux d'étude nationaux. Pour les Drom, la gestion de l'eau correspond à un secteur économique en pleine croissance demandant un personnel qualifié. La gestion de l'eau depuis la DCE représente ainsi une opportunité économique certaine pour ces territoires aux contextes socio-économiques fragiles.

Les réseaux de suivi des paramètres abiotiques et biotiques mis en place, dans le cadre de la DCE, fournissent les données actuellement exploitées pour connaître l'état des milieux. Ainsi, les futures séries statistiques historiques, dont les Drom ont été dépourvues, apporteront de nombreuses réponses, tout en faisant émerger d'autres problématiques. Si les listes taxonomiques étaient bien établies pour la macrofaune et la macroflore aquatiques d'eau douce et salée avant l'entrée en vigueur de la DCE, il en est bien différent pour les populations de taille plus petite, connues uniquement par de rares experts naturalistes. Dès lors, les inventaires et les connaissances acquises pour des essais d'indicateurs biologiques à partir de diatomées ou de macro-invertébrés n'ont, par exemple, débuté qu’à partir des années 1990 à la Martinique (Dauta et al., 1995; Dauta et Bosca, 1997; Klenklen et Thenard, 2000). Avec l'arrivée de la DCE, une nouvelle impulsion est venue relancer la recherche sur les indices biologiques, avec par exemple l'organisation d'un colloque sur la bio-indication en milieux aquatiques d'eau douce tropicale à Fort-de-France en 2010.

La DCE contribue à orienter la politique de l'eau tout en laissant aux territoires la capacité de se focaliser sur des problématiques spécifiques à leurs districts : extraction minière, agriculture d'exportation, exploitation touristique, risques sanitaires, etc. À travers les plans de gestion du Sdage, l'application de la DCE dans les Drom peut trouver un cadre d'application suffisamment approprié aux spécificités locales. Parallèlement, l'application de la directive a largement dynamisé l'acquisition de connais- 
sances en questionnant la nécessité de développer des protocoles standardisés homogènes et reproductibles. En revanche, une lecture territoriale plus approfondie interroge sur l'impossibilité d'accessions aux injonctions planifiées par la DCE.

\section{DES ÉLÉMENTS CONTEXTUELS CONTRAIGNANT L'APPLICATION DE LA DCE : ENTRE INCERTITUDE ET RISQUE}

\section{Un contexte socio-écologique propice à l'incertitude}

Le $\mathrm{xx}^{\mathrm{e}}$ siècle a été marqué par de profondes mutations des modes d'utilisation et d'occupation de l'espace dans les Drom. L'archipel de la Guadeloupe, la Guyane, la Martinique, l'île de Mayotte et la Réunion ont en effet connu une diversification des usages de l'espace. Bien que l'organisation spatiale actuelle renvoie à la distinction entre cultures de plantation, espaces naturels et zones urbanisées aux Antilles et à la Réunion, le développement d'un double processus de mitage et d'enfrichement de parcelles abandonnées, a donné lieu à une diversification paysagère propice à la multifonctionnalité des lieux. La situation guyanaise, a priori différente du fait de la faible densité de population et de la superficie du territoire, s'insère dans une dynamique aussi contraignante. En effet, la disponibilité foncière apparait comme étant un obstacle majeur au développement endogène, notamment pour l'agriculture. L'essentiel de l'occupation humaine se concentre sur la frange littorale et plus ponctuellement aux abords de fleuves. L'État possède près de $95 \%$ du territoire, consacrés pour l'essentiel à la conservation de la biodiversité et à l'exploitation des ressources forestières. Parallèlement, les zones propices au développement de l'urbanisation et de l'agriculture se trouvent aussi sous-contraintes du fait de la mise en protection d'espaces naturels.

La multifonctionnalité des lieux qui en résulte est propice à l'émergence d'une incertitude socioécologique. L'étude de la complexité des processus interactifs entre les systèmes sociaux et les systèmes écologiques, a été initiée en vue de comprendre les impacts des perturbations sur la résilience des écosystèmes et le maintien des services écosystémiques. Le concept de système socio-écologique
(Ses) a notamment été explicité par Fikret Berkes et Carl Folke en 1998, dans l'ouvrage Linking social and ecological systems. La socialisation de la nature, suggérée par cette approche des rapports des humains à leur milieu, renvoie à l'analogisme de la pensée « descolienne» (Descola, 2005). Le concept de Ses et son processus d'analyse régionale (Moran-Ordonez et al., 2013; De Aranzabal et al., 2008; Bourgeron et al., 2009) rappellent une nécessité d'inclure les compartiments sociaux et politiques dans la connaissance des écosystèmes, de leurs interactions et de leurs fonctions (Berkes et al., 2003). De récentes réflexions sur cette problématique ont notamment mis l'accent sur la capacité des institutions à maintenir des territoires dans un état de stabilité ou de créer des opportunités de changement pour l'obtention d'un autre état de stabilité (Janssen, 2011 ; Bourgeron et al., 2009). L'incertitude des savoirs scientifiques s'explique en partie par cette tendance de «la science moderne à l'étude de systèmes hétérogènes - écosystèmes, corps humain, économie mondiale et ainsi de suite -, qui sont à peu près aussi complexes que le système climatique et qui renferment chacun tant d'éléments et d'interactions potentielles qu'ils sont en pratique imprévisibles » (Hansson, 2002). Approchée sous le prisme de la socialisation de la nature, l'incertitude peut être interprétée comme une condition dans laquelle les non-intentionnalités d'une décision ne sont pas concevables a priori, mais uniquement constructibles a posteriori, notamment par réflexivité. Elle suggère donc que les individus et les organisations développent leurs capacités à la régulation des systèmes socioécologiques au travers d'une adaptabilité spatio-temporelle à l'irréversibilité des changements dans les systèmes écologiques. Les Drom connaissent une situation problématique de pollution à grande échelle caractérisée par une incertitude des savoirs relatifs à ses persistances et à ses impacts sanitaires : pesticides persistants dans l'environnement pour les Antilles, Mercure en Guyane, risques sanitaires concernant plusieurs bassins-versants dromiens par exemple.

Au-delà de la complexité du socioécosystème, l'efficacité des situations d'expertise est limitée du fait de fortes relations sociales de proximité entre les individus et les organisations en situation d'insularité. En 2002, Thierry Michalon fait le constat d'une forme de promiscuité spécifique aux Drom. 
Stéphane Gombaud (2007) généralisera la proposition aux espaces archipélagiques en expliquant que l'intrusion tardive des îles dans les logiques de l'économie de marché et de l'État moderne, est à l'origine d'un « dévoiement des règles démocratiques et d'une inégalité de traitement des citoyens par l'administration locale trop peu républicaine ». Dans son analyse des relations sociales comme frein ou moteur de la durabilité, Jean-Marc Callois (2006) précise les risques liés aux situations « de réseaux sociaux trop refermés sur eux-mêmes » : maintien difficile des performances économiques; diminution des incitations; effets de collusion. Bien que mal documentées, ces situations s'illustrent notamment par l'observation de transferts réciproques de personnels entre bureaux d'étude et administrations, ainsi que par des récurrences dans les attributions de marchés. Cela est notamment visible à la Martinique où il a été constaté que les expertises effectuées entre 2005 et 2013 sur les compartiments des eaux de surfaces, des eaux souterraines et des eaux de transition et marines, ont été respectivement réalisées par les mêmes bureaux d'étude ${ }^{3}$. L'expertise collective et pluridisciplinaire, dont l'importance dans tout le dispositif décisionnel démocratique n'est plus à démontrer (Lascoume, 2002), est souvent inexistante ou peu intégrée du fait de l'exiguïté et de la non-reconnaissance des communautés d'experts locaux. Cela s'illustre dans le thème de la continuité écologique. En effet, il est établi que l'on retrouve une population de poissons et de crevettes riche ( 3 Gobideaes, 4 Palaemonidaes et 2 Atyidaes) en amont d'une cascade de "plusieurs dizaines de mètres en verticale » (FDAAPPMA, 2011) dans la rivière Duclos (Martinique), et « des populations importantes d'Atya innocous " (Tito de Morais et al., 1993) en amont de la deuxième chute de la Rivière du Carbet (Guadeloupe) d'une hauteur supérieure à 110 m, à une altitude de 890 m. En 2007, il fut même reconnu «que les seuils, les chutes naturelles sur le lit ne viennent pas réellement perturber la distribution des espèces » (ODE-DIREN, 2008). Et pourtant, sans considérer ces savoirs, les études d'impacts concernant la continuité écologique, centrées sur les obstacles à la migration en milieu torrentiel, se multiplient, notamment à la Martinique, pour étudier des seuils de quelques

3. [www.observatoire-eau-martinique.fr]. mètres de hauteur de chute tout au plus (Dal Pos, 2010 ; CAP Nord Martinique, 2014; SICSM, sous presse; ODE, sous presse). L'intérêt scientifique de ces études devient alors difficile à établir, car, hormis les prises d'eau, seuls les obstacles des plaines alluviales (habitat lentique) impactent réellement la migration des espèces non adaptées au franchissement. Cet exemple illustre une application en accord avec l'esprit de la DCE qui, pourtant, ne répond pas aux exigences des Drom insulaires.

De même, en Guyane, il apparaît très clairement que les inondations catastrophiques de 2006 et 2008, avec au total plus d'un millier de personnes déplacées, de nombreuses habitations et commerces touchés et une victime, questionnent la fabrique de dispositifs institutionnels qui intègrent les savoirs existants relatifs aux risques naturels. Malgré le Plan de prévention des risques naturels (PPRN) de 1997, puis les Plans de prévention du risque d'inondation (PPRI) communaux des années 2000, le fleuve Maroni demeurait toujours un angle mort de la politique gestion du risque d'inondation. En 2005, avec l'Atlas des zones inondables (AZI), le document s'intéressait bien à la menace d'inondation sur le Maroni, mais en se focalisant uniquement sur deux communes à l'aval : Saint-Laurent et Apatou. Ce n'est donc qu'à la suite des inondations catastrophiques, que le Plan de gestion du risque d'inondation (PGRI) de la Guyane, qui devrait être opérationnel en 2015, intègre l'ensemble des communes du fleuve Maroni.

Plus encore, le retard de développement commun aux cinq Drom est aussi un retard dans la production de connaissances sur l'environnement. Les réseaux de mesures abiotiques et les inventaires biocénotiques, dont la pression d'échantillonnage est acceptable, ne datent que des années 1990. Les séries statistiques sont donc relativement pauvres et peu représentatives dans le temps. Une expertise réalisée sur quatre années, dans 86 stations ayant fait l'objet de prélèvements saisonniers, a produit 470 lots d'analyse (ODE-DEAL-ONEMA, 2009, 2011 a, 2011b, 2012; DEAL, 2010). Malgré cette apparente forte pression d'échantillonnage, il n'est actuellement pas possible d'estimer la contamination des animaux dans certains compartiments, plus particulièrement des juvéniles aux embouchures, ainsi que leur décontamination naturelle en Chlordécone lors de leur migration en eaux saines. 
De plus, compte tenu d'un ensemble de facteurs aggravants comme l'intensité des précipitations tropicales, les reliefs accidentés, l'exigüité des bassins-versants et le mitage du paysage, les concentrations de polluants sont particulièrement variables dans l'espace, mais aussi et surtout dans le temps (Mottes, 2013). Dès lors, une surveillance ponctuelle de la physicochimie des eaux de surface courantes ne permet pas d'appréhender l'état réel du milieu. L'inadaptation des prélèvements ponctuels à la physico-chimie pose d'ailleurs la question de la non-représentativité temporelle soulignée par Gueguen et al. (2013) d'indices biologiques développés dans le cadre de la DCE sur la base de séries statistiques qui ne concernent que trop peu d'années. Ainsi, que ce soit pour les diatomées (Gueguen et al., 2013) ou les macro-invertébrés (Bernadet et al., 2013), la pertinence des indices biologiques a dû davantage s'appuyer sur les caractéristiques de la population présente, que sur la sensibilité aux polluants des individus constituants cette population. Cette approche, imposée du fait des incertitudes des savoirs, demeure cependant dans l'esprit de la DCE, celle d'une évaluation de l'état écologique, exprimée en termes d'écart par rapport aux conditions dites de référence, Ecological quality Ratio (EQR).

Enfin, les délais particulièrement courts de mise en place des protocoles d'acquisition de données, n'ont pas favorisé une réflexion concertée suffisamment efficiente pour répondre aux problèmes posés à des fins de mise en œuvre d'actions. Par exemple, l'intégration limitée des expériences sur les questions de l'eau, indispensable pour des actions menées sur le terrain, ne contribue pas à l'efficience des conclusions des expertises. À la Martinique, il n'existe pas d'inventaire et de suivi faunistique exhaustifs pour les eaux de transition, les étangs et les mares. Or, une écrevisse invasive (Cherax quadricarinatus), importée pour l'aquaculture il y a moins de 10 ans, est actuellement en phase de colonisation des plans d'eau et des cours d'eau du territoire. Ces situations sont d'autant plus favorables au développement de controverses confortées par les incertitudes accrues du fait de la multifonctionnalité des lieux.

\section{Un contexte environnemental propice à l'exacerbation des risques}

En s'appuyant sur les écrits de Franck Knight (1921) relatifs aux notions de risque et d'incertitude,
Douglass North (1990) apporte une piste d'explication à ce problème. Il suggère que les termes de risque et d'incertitude ont subi une forme d'altération sémantique conduisant à désigner le risque comme une incertitude (North, 2005). Or, si le risque suppose une condition dans laquelle il est possible de calculer une distribution probable des résultats, l'incertitude par contre ne dispose pas de cette information probabiliste.

Dans le cas des Drom, ils possèdent la particularité d'être soumis à un ensemble d'aléas naturels accroissant la vulnérabilité des écosystèmes des petits territoires. Les menaces naturelles, particulièrement nombreuses, sont d'origines hydroclimatiques (cyclones, inondations, sécheresses) et géodynamiques (séismes, éruptions volcaniques, mouvements de terrain, tsunamis). Elles ponctuent l'histoire de ces îles de grandes catastrophes, mettant en lumière les capacités de résilience des systèmes anthropiques et naturels de ces territoires. En Guyane, les menaces naturelles sont représentées essentiellement par les inondations, les submersions marines et les mouvements de terrain.

Au-delà de ces risques « dits » naturels (Pingeon, 2005; Léone et al., 2010), de nombreux types de risques sanitaires sont également étroitement liés à l'eau. Dans certaines communautés de populations dromiennes, les tâches domestiques et sanitaires se font couramment dans les rivières. La fréquentation, mêlée aux usages non conformes aux règles sanitaires actuelles, génère des problèmes d'hygiène et de santé. Aux Antilles, le risque de contamination à grande échelle, disparu au cours des années 1980 avec l'arrêt de la transmission de la Bilharziose (Pointier et Guyard, 1992; Pointier et al., 2011), a été réactivé par la découverte d'un empoisonnement de grande ampleur à un pesticide, la Chlordécone (Snégaroff, 1977), un insecticide utilisé pour lutter contre le charançon (Cosmopolites sorditus) du bananier. Du fait des spécificités de la faune locale, ce risque est d'autant plus accru dans les Drom insulaires. En effet, la faune aquatique d'eau douce a conservé une certaine tolérance à la salinité, critère majeur d'adaptation en milieu insulaire (Keith et al., 1999). De plus, les conditions torrentielles et l'exiguïté des bassins-versants limitent très fortement le développement du plancton, pouvant alimenter les larves de poissons ou de crustacés. L'évolution a donc favorisé des taxa pour lesquels le dévelop- 
pement larvaire s'effectue en milieux marins salins jusqu'au stade juvénile : il s'agit de la diadromie. Si certains taxa pondent en rivière et après éclosion les larves dévalent passivement avec le courant jusqu’à la mer (amphidromie), d'autres pondent en mer ou en eau saumâtre (catadromie) avant de revenir dans le cours d'eau. Quel que soit le procédé, la faune dulçaquicole des Drom insulaires se renouvelle uniquement depuis l'aval (figure 2 - planche II), dans une migration à contre-courant en direction des zones torrentielles, par rhéotactisme positif. L'embouchure représente donc un goulet d'étranglement dans le cycle biologique des espèces. Et pourtant, elle se situe dans la zone la plus anthropisée du bassin versant et subit de ce fait de très fortes pressions polluantes : artificialisation du lit contre les crues, rejets urbains, ruissellement pluvial des surfaces imperméabilisées, pollutions agricoles, etc. Les quatorze espèces de crustacés décapodes sont les mêmes en Martinique et en Guadeloupe. En outre, 21 et 16 espèces de poissons ont été respectivement recensées en Martinique et en Guadeloupe (Lim et al., 2002; Monti et al., 2010). Bien que les espèces de la Réunion soient différentes de celles des Petites Antilles, elles appartiennent aux mêmes familles taxonomiques (Keith et al., 1999). Ce processus migratoire continuel de l'aval vers l'amont suppose que les animaux contaminés diffusent la pollution vers les milieux naturels des espaces forestiers et montagneux (figure 3 - planche III). La chaîne trophique et les filières de décomposition participent dès lors à la diffusion spatiale de pollutions agricoles dans les milieux naturels aquatiques éloignés de sites contaminés (Coat, 2009). Les étages bioclimatiques, qui organisent les territoires insulaires, ne constituent pas un rempart aux échanges biophysiques entre l'aval et l'amont des bassins-versants. Ainsi, la moitié supérieure des bassins-versants (au-delà de 500 m d'altitude environ) qui théoriquement devrait être préservée de toute pression polluante ne correspond finalement pas à une réalité.

Ces particularités sont nécessairement à prendre en compte dans la gestion des milieux aquatiques. Cependant, l'exiguïté, l'isolement ainsi que la fragilité institutionnelle, écosystémique, sociétale et économique de ces territoires, les exposent à des éléments contextuels particuliers pouvant contraindre l'application de la DCE.

\section{LES DIFFICULTÉS D'APPLICABILITÉ DE LA DCE : LE CAS DE LA MARTinique}

\section{Un contexte d'incertitudes des savoirs technico-scientifiques}

Les terres agricoles des Antilles françaises sont actuellement contaminées par des pesticides persistants dans l'environnement. La catastrophe de la contamination à la Chlordécone est d'autant plus accentuée aux Antilles, que cette molécule est persistante dans l'environnement (Cabidoche et al., 2008). Par les processus de bioconcentration des individus et de bioaccumulation au sein du réseau trophique, la faune aquatique présente des concentrations plus de 1000 fois supérieures à une norme sanitaire de $20 \mu \mathrm{g} / \mathrm{kg}$ de poids frais. En lien avec l'érosion agricole (Cabidoche et al., 2008) et les eaux d'infiltrations (Cattan et al., 2012), les cours d'eau, leurs chaines trophiques et les milieux marins en aval sont le réceptacle de ces contaminants. Avec un lessivage, dont la durée peut s'estimer en siècle selon le type de sol (Cabidoche et al., 2008), les compartiments des milieux aquatiques subiront la contamination de la Chlordécone. Si les pistes de recherche sont encourageantes, les techniques de remédiation à l'échelle des parcelles agricoles ne seront probablement pas mises en œuvre à l'échéance 2027 (Lesueur-Jannoyer et al., 2011 ; Godard, 2012). Au regard de la persistance de ces pesticides dans l'environnement, les milieux aquatiques n'atteindront pas les critères de bon état, attendus dans les délais impartis par la DCE.

De plus, comme évoquées précédemment, les connaissances scientifiques relatives aux milieux aquatiques sont encore trop parcellaires. L'implication trop limitée des universités et des organismes de recherche sur ces questions de la gestion de l'eau à la Martinique, justifie la succession de missions spontanées ou d'études ponctuelles qui, bien que de qualité, ne répondent pas toujours aux critères d'homogénéité et de reproductibilité de la démarche scientifique. Seuls de récents inventaires hydro-morphologiques, comme le protocole CARHYCE développé par l'Office national de l'eau et des milieux aquatiques, l'Onema (DEAL, 2012), ainsi qu'un certain nombre de réseaux de surveillance des milieux, exprimant la volonté des Sdage 
dans le cadre de la DCE, répondent depuis peu à ces impératifs scientifiques. Dans les conditions d'un manque de recul sur l'information scientifique acquise, les experts courent le risque de formuler des mesures de gestion et des critères d'évaluation non adaptés. Plus encore, la représentativité limitée des discours de la contre-expertise favorise le développement de contestations justifiées autour des résultats acquis. De nombreuses associations de défense de l'environnement jouent ce rôle en émettant parfois de vigoureuses réserves sur les décisions prises par les gestionnaires. Cela s'illustre notamment par l'absence de certitudes concernant la contamination de la faune d'eau douce en altitude, ou plus encore par la mise en œuvre de plusieurs protocoles de prélèvements biologiques dans une même ravine sèche (ODE-DEAL-ONEMA, 2011 b; DEAL-ODE, 2012). De même, le protocole de pêche électrique appliqué jusqu'en 2009 (DIREN, 2010) a été modifié en faveur de la méthode d'Échantillonnage ponctuelle d'abondance (EPA), mise au point par l'Onema dans le cadre du réseau de surveillance DCE pour les rivières supérieures à huit mètres ou dont la prospection à pied est impossible ${ }^{4}$. Pourtant, des essais en Guadeloupe ont démontré que cette méthode n'est pas adaptée pour l'estimation des stocks vivants dans les cours d'eau des Antilles (Fiévet et al., 1996). Il est en plus précisé que la méthode d'EPA a été " adaptée au contexte tropical » (DIREN, 2010) en raison d'une « forte densité d'individus » (DEALODE-ONEMA, 2011 a), pour réduire la longueur pêchée et réduire la surface de pêche en ne déplaçant pas l'anode (DEAL-ODE-ONEMA, $2011 \mathrm{a}$ ). Or, cette affirmation d'une surabondante densité de population est en contradiction avec le constat que durant cette même période les « efforts de pêche n'ont pas permis de constituer certains lots de poissons ou de crustacés » pour l'analyse de la Chlordécone dans les chairs (ODE-DEAL-ONEMA, 2012). Faute de véracité scientifique, la gestion de l'eau dans les Drom peut se transformer insidieusement en une transposition des concepts hydrobiologiques continentaux tempérés, aussi bien de la part des acteurs qualifiés «d'expatriés » par les dromiens, que des acteurs dromiens formés en métropole. Quand cette tendance s'affirme, les mesures de gestions

4. Norme NF EN 14011, protocole XP T90-383 de mai 2008. entreprises peuvent devenir préjudiciables pour les milieux aquatiques locaux, tout en donnant l'illusion de répondre aux exigences exprimées dans la DCE. Ces contextes d'incertitudes des savoirs technicoscientifiques, qui caractérisent l'ensemble des Drom sur des thématiques spécifiques aux territoires, limitent la capacité de la Martinique de répondre aux injonctions d'agenda de la DCE.

\section{L’inertie du principe de précaution}

Les organisations locales et nationales en charge de la gestion des eaux à la Martinique adoptent des attitudes prudentes face à des initiatives d'actions concrètes de protection des eaux. L'exemple de la proposition de classement des cours d'eau sur « la liste 1 » des réservoirs biologiques ${ }^{5}$ illustre ce constat. Seuls les cours d'eau pressentis par les rédacteurs du Sdage en 2009 ont été retenus ${ }^{6}$ : les autres critères prévus dans le même l'article du code, le «bon état écologique » et la «protection des migrateurs » (DEAL, 2014), n'ont pas été pris en compte dans la démarche de classement. Au regard des connaissances produites (DEAL-ODE, 2012), le choix des cours d'eau classés au titre de réservoirs biologiques s'est révélé incohérent à cause de constats de prises d'eau à l'aval, d'assèchement intermittent du débit avant l'embouchure et de rejets d'eau de mer utilisée pour le refroidissement d'une usine thermique. Cette situation renvoie à une forme d'inertie légitimée par une norme de l'action publique, le principe de précaution (Godard, 2003). Du point de vue de la doctrine institutionnelle, le principe de précaution réfère à des situations d'absence de certitudes démontrées et de fortes présomptions de risques. Dans son acception précautionneuse, le principe de précaution traduit une prise en compte de la non- ergodicité du monde en réponse aux incertitudes - voire même à l'ignorance - inhérentes à la mise en œuvre d'actions publiques.

Plus encore, il apparaît ainsi que, malgré de nombreuses tentatives depuis le début du XVIII ${ }^{\mathrm{e}}$ siècle (FDAAPPMA, 2011), la pêche en rivière n'a jamais pu être réglementée à la Martinique. La Fédération départementale des associations agréées de pêche et de protection des milieux aquatiques (FDAAPPMA) de la Martinique milite pour la gestion de ces

5. Art. L.214-17 du code de l'environnement. 6. Arrêté 2015022-0006 du 22 janvier 2015. 
milieux d'eau douce, avec le soutien de la Direction de l'environnement, de l'aménagement et du logement (Deal), du Conseil régional et de l'Office de l'eau (ODE). Elle a notamment initié la réalisation d'un atlas de la faune d'eau douce (Lim et al., 2002) et l'élaboration d'un schéma départemental de vocation piscicole (FDAAPPMA, 2011, 2013). Malgré la contamination de la faune d'eau douce par la Chlordécone sur environ les deux tiers du territoire (ODE-DEALONEMA, 2012), la pêche pourrait encore être pratiquée dans la région nord-caraïbe, avec certaines prescriptions (FDAAPPMA, 2011). Dans le contexte actuel de fermeture totale de la pêche due à la contamination de la faune ${ }^{7}$, il paraît illusoire de croire que la fédération de pêche pourra organiser, mettre en place et pérenniser un système de droit de pêche. Malgré une volonté partagée de la part des pêcheurs, des gestionnaires et de la fédération de pêche de la Martinique, il semble que la réouverture de la pêche en rivière soit des plus insurmontables du fait de son manque de professionnalisation.

Cet exemple illustre la problématique des imprévisibilités de la régionalisation de l'État dans le cadre de la révision générale des politiques publiques (Le Bourhis et Martinais, 2014). En effet, le renversement du « jacobinisme administratif » se heurte à une véritable inertie des instances en charge de la question de la pêche en rivière à la Martinique, probablement liée à une attitude précautionneuse des agents déconcentrées de l'État, justifiée par les ignorances, les incertitudes et les risques qui alimentent un climat social tendu autour de la question de la contamination par la Chlordécone (Verdol, 2014). Les réponses apportées par les modèles classiques de territorialisation de l'action publique (Reliant, 2004) favorisent la controverse et l'inertie. Le modèle décisionniste qui repose sur l'expertise ne peut s'avérer pertinent dans ses situations «dromiènes » de multifonctionnalité de lieux, propice à l'incertitude, au risque et à la controverse. Du fait de ses caractères normatifs et impératifs, le cadrage descendant de la DCE oblige les régions à la mise en ouvre des forums hybrides qui contrarient les inerties de l'action collective en favorisant l'augmentation des incitations et en réduisant les effets de collusion. Ainsi, la gouvernance devrait

7. Arrêté 09-03540 du 25 septembre 2009, reconduit annuellement. s'envisager par l'adoption de mesures en se référant à une démarche délibérative porteuse d'expertises collectives et pluridisciplinaires propres au modèle pragmatique de territorialisation de l'action publique (Reliant, 2004).

\section{Conclusion}

Si l'application de la DCE dans les Drom a effectivement été porteuse d'avancées notables en imposant aux gestionnaires un effort soutenu de concertation territoriale et d'acquisition de connaissances scientifiques, l'insuffisance de données scientifiques historiques ne permet pas de définir des « conditions de référence ", dont la construction s'appuierait sur une approche métastable de l'équilibre des écosystèmes. Dans cette conception, les perturbations passées, y compris celles qui sont d'origine anthropique, tendent à influer de façon irréversible leurs dynamiques. Loin d'être unique dans les régions de l'Union européenne (Porcher, 2009), l'exemple de la Martinique illustre les difficultés que rencontrent les Départements et Régions d'outre-mer à donner sens au concept de "conditions de référence », difficilement définissable du point de vue de l'état chimique (Quevauviller, 2009) à cause d'incertitudes dans les savoirs technico-scientifiques. Les contextes problématiques de pollutions récentes des milieux aquatiques constituent en plus une contrainte difficilement surmontable pour atteindre l'injonction de ce «bon état écologique » dans le respect des dates butoirs prescrites par l'Union européenne. L'échéancier et les reports autorisés par la DCE ne sont pas viables dans les Drom du fait de connaissances encore trop incertaines qui rendent difficiles la conception de seuillages quantitatifs efficients. Au-delà de la question des conditions de référence, l'inertie autour de la mise en œuvre d'actions concrètes de gestion durable des systèmes aquatiques est d'autant plus exacerbée dans ces territoires. Les situations conjoncturelles où incertitude des savoirs, risque et principe de précaution s'entremêlent accroissent les durées de mise en ouvre de l'action collective.

Les bilans d'actions et les remontées administratives quantitatives, imposés par l'État et les instances de l'Union européen, traduisent une apparente congruence entre les injonctions de la DCE et les Sdage dromiens. Si les évaluations des résul- 
tats intrinsèques des politiques publiques relatives à la gestion de la ressource en eau dans les Drom peuvent donner cette impression, la question des évaluations extrinsinques, qui jugent de la valeur de la politique en analysant son impact sur l'évolution du développement durable (Ruprich-Robert et Bencivenga, 2002), suscite de vives interrogations dans ces contextes. Rappelons que le Conseil scientifique de l'évaluation définit la démarche évaluative comme étant un processus visant à « appréhender d'un point de vue principalement externe les effets et/ou la valeur d'une action considérée » (CSE, 1997). Les évaluations concomitantes prévues en 2015 et en 2021 devraient être révélatrices de ces discordances entre les conceptions de la DCE en matière de gestion des systèmes aquatiques et les possibilités dont disposent réellement la Guadeloupe, la Guyane, la Martinique, Mayotte et la Réunion. En prévention, les organisations compétentes sur la gestion des milieux aquatiques dans les Drom gagneraient à investir collectivement les instruments financiers comme le programme Life. Ils dynamiseraient la mise en œuvre d'actions concrètes de préservation et d'amélioration de l'état écologique des systèmes aquatiques, à partir de « conditions de référence » notamment instituées selon les valeurs socio-écologiques des systèmes aquatiques.

\section{Bibliographie}

Berkes F., Colding J., Folke C., 2003. Navigating social-ecological systems: building resilience for complexity and change, Cambridge University Press, Cambridge, 416 p.

Berkes F., Folke C., 1998. Linking social and ecological systems for resilience and sustainability, in Berkes F., Folke C., Colding J. (dir.), Linking social and ecological systems: management practices and social mechanisms for building resilience, Cambridge, Cambridge University Press, p. 1-25.

Bernadet C., Bargier N., Cereghino R., 2013. Mise au point d'un indice de bioindication de la qualité de l'eau des cours d'eau de Martinique à partir des macroinvertébrés benthiques, Rapport ODE972, DEAL972, ONEMA, ASCONIT Consultants, Fort-de-France, 62 p. [http://www observatoireeau-martinique.fr/les-outils/base-documentaire/tris-base-documentaire/suivi-des-milieux-aquatiques].

Bouleau G., Pont D., 2014. Les conditions de référence de la directive cadre européenne sur l'eau face à la dynamique des hydrosystèmes et des usages, Natures, Sciences et Sociétés, 22, (1), 3-14. [http://dx.doi.org/10.1051/ nss/2014016].
Bourgeron P., Humphrie H., Riboli-Sasco L., 2009. Regional Analysis of Social-Ecological Systems, Natures sciences sociétés, vol. 17, n² 2, p. 185-193.

Cabidoche Y.M., Clermont-Dauphun C., Caron A., Cattant P., Chabrier C., Lafont A., Lesueur-Jannoyer, SansouLET J., 2008. Stockage dans les sols et dissipation dans les eaux de la chlodécone, insecticide organochloré autrefois appliqué dans les bananeraies des Antilles Françaises, Les cahiers du Pôle de Recherche Agro-environnementale de la Martinique, $\mathrm{n}^{\circ}$ 7, p. 39-43.

Callois J.-M., 2006. Les relations sociales, frein ou moteur de la durabilité, Développement durable et territoires [En ligne], dossier 8, [http://dx.doi.org/10.4000/developpementdurable.3284].

CAP Nord Martinique, 2014. Rivière du Galion : inventaire hydro-biologique au niveau des principaux obstacles à la continuité écologique, rapport de Phase 3, Rapport Contrat de Rivière du Galion, Le-Marigot (Martinique), 74 p.

Cattan P., Cabidoche Y.-M., Colin F., Fernandez-Bayo J., Saison C., Voltz M., 2012. Des parcelles aux rivières : Comment la Chlordécone contamine-t-elle les eaux?, in Godard E., Les journées recherche du plan chlordécone, Recherches agro-environnementales et état des milieux, Dossier de présentation, 24-25 octobre 2012 Schølcher (Martinique) / Gosier (Guadeloupe), 31 p.

CоAт S., 2009. Identification du réseau trophique de rivière et étude de sa contamination par les pesticides organochlorés (Chlordécone et $\beta$-HCH) en Guadeloupe. Thèse de doctorat en écotoxicologie et écologie aquatique, université Antilles Guyane, 209 p.

CSE, 1997. Petit guide de l'évaluation des politiques publiques, Bulletin des bibliothèques de France [en ligne], Conseil scientifique de l'évaluation des politiques publiques.

Dal Pos N., 2010. Étude de la continuité écologique des cours d'eau de la Martinique, Master, université de Perpignan, $30 \mathrm{p}$.

Dauta A., Bosca C., Lim P., Dauba F., 1995. Étude floristique et faunistique des rivières martiniquaises, Diren, Fort de France, $73 \mathrm{p}$.

Dauta A., Bosca C., 1997. Inventaire de la faune benthique des cours d'eau de la Martinique; mise au point d'indice biotique, Diren, Fort-de-France, 109 p.

DEAL, 2010. Détermination de la contamination des milieux aquatiques par le chlordécone, les Titiris, Rapport Plan d'Action Chlordécone 2008-2010, Fort-de-France, 10 p. [http:// www.observatoire-eau-martinique.fr/les-outils/base-documentaire/tris-base-documentaire/suivi-des-milieux-aquatiques].

DEAL, 2014. Le classement des cours d'eau au titre de l'article L.214-17 du code de l'environnement, résumé non technique, Rapport, Fort-de-France, $10 \mathrm{p}$.

DEAL, 2012. CARHYCE, application du protocole aux rivières de la Martinique, Rapport, Fort-de-France, 39 p. [http:// www.observatoire-eau-martinique.fr/les-outils/base-documentaire/tris-base-documentaire/suivi-des-milieux-aquatiques].

DEAL-ODE, 2012. Étude de l'impact du classement des cours d'eau au titre de l'article L.214-17 du code de l'Environnement, synthèse hydrobiologique, Rapport, Fort-de-France, 65 p. [http:// 
www.observatoire-eau-martinique.fr/les-outils/base-documentaire/tris-base-documentaire/suivi-des-milieux-aquatiques].

De Aranzabal I., Schmitz M. F., Aguilera P., Pineda F. D., 2008. Modelling of landscape changes derived from the dynamics of socio-ecological systems: a case of study in a semiarid mediterranean landscape, Ecological Indicators, vol. $8, n^{\circ} 5$, p. 672- 685 .

Descola P., 2011. L'écologie des autres, Versailles, Quae Éditions, $106 \mathrm{p}$.

DIREN, 2010. Réseau de contrôle de surveillance, contrôle opérationnel et contrôle d'enquête des cours d'eau de la Martinique - Année 2009, volet Biologique, Rapport Final, Rapport, Fort-de-France, 99 p. [http://www.observatoire-eau-martinique.fr/les-outils/base-documentaire/tris-base-documentaire/ suivi-des-milieux-aquatiques].

FDAAPPMA, 2011. Diagnostic, les potentialités piscicoles des milieux d'eau douce de la Martinique, Rapport SDVP tome 2, Fort-de-France, $85 \mathrm{p}$.

FDAAPPMA, 2013. Objectifs et orientations, la reconquête des milieux aquatiques martiniquais : la réouverture de la pêche face à la contamination à grande échelle par la Chlordécone, Rapport SDVP, Tome 4, Fort-de-France, 56 p.

Fièvet E., Tito de Morais L., Tito de Morais A., 1996. Quantitative sampling of freshwater shrimps: comparaison of two electrofishing procedures in a Caribbean stream, Arch. Hydrobiol., vol. 138, n 2, p. 273-287.

Godard O., 2003. Le principe de précaution comme norme de l'action publique, ou la proportionnalité en question, Revue économique, vol. 54, $\mathrm{n}^{\circ}$ 6, p. 1245-1276.

Godard E., 2012. Les journées recherche du plan chlordécone, Recherches agro-environnementales et état des milieux, 24-25 octobre 2012 Schœlcher (Martinique)/Gosier (Guadeloupe), Dossier de présentation, 135 p.

Gombaud S., 2007. Îles, insularité et îléité, le relativisme dans l'étude des espaces archipélagiques. Thèse de doctorat en géographie, université de la Réunion, 1101 p.

Gueguen J., Eulin A., Lefrançois E., Boutry S., Rosebery J., Coste M., Delmas F., 2013. Mise au point d'un indice de bioindication de la qualité de l'eau des cours d'eau antillais à partir des diatomées : l'IDA, Rapport ODE972, DEAL972, DEAL971, ODE971, ONEMA, ASCONIT Consultants, Irstea, Pointe-à-Pitre, 190 p. [http://www.observatoire-eaumartinique.fr/les-outils/base-documentaire/tris-base-documentaire/suivi-des-milieux-aquatiques].

Hansson S.O., 2002. Les incertitudes de la société du savoir, Revue internationale des sciences sociales, vol. $1, \mathrm{n}^{\circ} 171$, p. 43-51.

Janssen M. A., 2011. Resilience and adaptation in the governance of social ecological systems, International Journal of the Commons, vol. 5, $\mathrm{n}^{\circ} 2$, p. 340-345.

Keith P., Vigneux E., Bosc P., 1999. Atlas des poissons et des crustacés d'eau douce de la Réunion, Paris, Muséum National d'Histoire Naturelle de Paris, coll. «Patrimoines Naturels », $\mathrm{n}^{\circ} 39,136 \mathrm{p}$.

Klenklen S., Thenard V., 2000 . Aide à la détermination des macro-invertébrés d'eau douce de Martinique, Fort-deFrance, Diren, 66 p.
Knight F.H., 1921. Risk, Uncertainty, and Profit, New York, Houghton Mifflin and Co, $381 \mathrm{p}$.

Lascoumes P., 2002. L'expertise, de la recherche d'une action rationnelle à la démocratisation des connaissances et des choix, Revue française d'administration publique, vol. 103, $n^{\circ} 3,369-377$.

Le Bourhis J.-P., Martinais E., 2014. Quelle architecture institutionnelle pour le développement durable? La restructuration des services régionaux du ministère de l'écologie, Revue française d'administration publique, $\mathrm{n}^{\circ} 149$, p. 223237.

Léone F., Meschinet de Richemond, Vinet F., 2010. Aléas naturels et gestion des risques, Paris, PUF, 288 p.

Lesueur-Jannoyer M. (Coor.), 2011. Remédiation à la pollution par la chlordécone aux Antilles, Les cahiers du Pôle de Recherche Agro-environnementale de la Martinique, 9-10, $98 \mathrm{p}$.

Lim P., Meunier F.-J., Keith P., NoëL P.-Y., 2002. Atlas des poissons et des crustacés d'eau donce de la Martinique, Paris, Muséum National d'Histoire Naturelle de Paris, coll. «Patrimoines Naturels; 51 », 120 p.

Magnan A., 2009. La vulnérabilité des territoires littoraux au changement climatique : mise au point conceptuelle et facteurs d'influence, Institut du développement durable et des relations internationales, Paris, collection Analyse, 29 p.

Michalon T., 2002. Sur les "spécificités » de l'Outre-mer : enquête et propositions, Hermès, La Revue, vol. 1, n 32-33, p. 423-434

Monti D., Keith P., Vigneux E., 2010. Atlas des poissons et des crustacés d'eau douce de la Guadeloupe, Paris, Muséum National d'Histoire Naturelle de Paris, coll. « Patrimoines Naturels ", n 69. 128 p.

Moran-Ordonez A., Bugter R., Suarez-Seoane S., de Luis E., Calvo L., 2013. Temporal changes in socio-ecological systems and their impact on ecosystem services at different governance scales: a case study of heathlands, Ecosystems, vol. $16, n^{\circ} 5$, p. $765-782$.

Motтes C., 2013. Evaluation des effets des systèmes de culture sur l'exposition aux pesticides des eaux à l'exutoire d'un bassin versant. Proposition d'une méthodologie d'analyse appliquée au cas de l'horticulture en Martinique. Thèse de doctorat en sciences agronomiques, Institut des sciences et industrie du vivant et de l'environnement, 209 p.

North D.C., 1990. Institutions, institutional change and economic performance, Cambridge, Cambridge University Press, $159 \mathrm{p}$.

North D.C., 2005. Le processus du développement économique, Paris, Éditions d'Organisation, 237 p.

ODE, sous presse. Diagnostic de la continuité écologique de douze obstacles à la migration des espèces d'eau douce à la Martinique, Rapport d'étude.

ODE-DEAL-ONEMA, 2009, Détermination de la contamination des milieux aquatiques par le chlordécone et les organochlorés, rapport final, Rapport Plan d'Action Chlordécone 2008-2010, Fort-de-France, 88 p. [http://www.observatoireeau-martinique.fr/les-outils/base-documentaire/tris-base-documentaire/suivi-des-milieux-aquatiques]. 
ODE-DEAL-ONEMA, 201 la, Détermination de la contamination des milieux aquatiques par le chlordécone, volet 2 , investigations complémentaires, nouvelles zones et réévaluation, Rapport Plan d'Action Chlordécone 2008-2010, Fortde-France, 28 p. [http://www.observatoire-eau-martinique. fr/ les-outils/base-documentaire/tris-base-documentaire/suivi-desmilieux-aquatiques].

ODE-DEAL-ONEMA, 2011 b. Détermination de la contamination des milieux aquatiques par le chlordécone, Volet 3, investigations complémentaires, Têtes de bassins-versants et Sud Martinique, Rapport Plan d'Action Chlordécone 20082010, Fort-de-France, 55 p. [http://www.observatoire-eaumartinique.fr/les-outils/base-documentaire/tris-base-documentaire/suivi-des-milieux-aquatiques].

ODE-DEAL-ONEMA, 2012. Détermination de la contamination des milieux aquatiques par le chlordécone, Volet 4, investigations complémentaires, renforcement $d u$ maillage géographique sur les cours d'eau d'intérêt piscicole, Rapport Plan d'Action Chlordécone 2008-2010, Fort-de-France, 37 p. [http://www.observatoire-eau-martinique.fr/les-outils/ base-documentaire/tris-base-documentaire/suivi-des-milieuxaquatiques].

ODE-DIREN, 2008. État des lieux de l'environnement piscicole de la Martinique, Phase 2, diagnostic et potentialités piscicoles, Rapport ODE-DIREN, Fort-de-France, 111 p. [http:// www.observatoire-eau-martinique.fr/les-outils/base-documentaire/tris-base-documentaire/suivi-des-milieux-aquatiques].

Pigeon P., 2005. Géographie critique des risques, Paris, Economica, 217 p.

Pointier J.P., Guyard A., 1992. Biological control of the snail intermediate hosts of Schistosoma mansini in Martinique, French West Indies, Tropical Medecine and Parasitology, vol. 43, n², 98-101.

Pointier J.P., David P., Jarne P., 2011. The biological control of the snail hosts of Schistosomes: the role of competitor snails and biological invasions, in Toledo R., Fried B. (dir.), Biomphalaria snails and larval trematodes, New York, Springer, p. 215-238.

Porcher J.-P., 2009. Le cadre proposé par la Directive Cadre Européenne sur l'eau : outils de mesure et évaluation de l'état des eaux, La Houille Blanche, n 3, p. 41-45.

Quevauviller P., 2009. Evaluation de l'état chimique des eaux de surface et souterraines au titre de la directive cadre sur l'eau - normes de qualité et surveillance, La Houille Blanche, $\mathrm{n}^{\circ} 4$, p. 72-76.

Reliant C., 2004. L'expertise comme outil de territorialisation d'une politique publique? Fonctions et usages de l'expertise socio-économique dans la politique d'aménagement des zones inondables en France et en Angleterre. Thèse de doctorat en sciences et techniques de l'environnement, École nationale des ponts et chaussées, $472 \mathrm{p}$.

Ruprich-Robert C., Bencivenga M., 2002. Évaluation des politiques publiques : nouveau guide pratique, La lettre du cadre territorial, Voiron, 181 p.

SICSM, sous presse. Rétablissement de la continuité écologique de la Rivière Blanche et de la Rivière Lézarde et rectification des gués, Rivière Salée (Martinique), Rapport d'étude.

SNÉGAROFF J., 1977. Les résidus d'insecticides organochlorés dans les sols et les rivières de la région bananière de Guadeloupe. Phytiatrie-Phytopharmacie, 26, p. 251-268.

Tito de Morais L., Monti D., Rambaud V., Lauzanne L., 1993. Étude hydrobiologique de la Rivière du Grand Carbet, Basse-Terre, Pointe-à-Pitre, Guadeloupe, Rapport ORSTOM, $68 \mathrm{p}$

Verdol P., 2014. Du chlordécone comme arme chimique française en Guadeloupe et en Martinique, et de ses effets en Europe et dans le monde, Paris, L'Harmattan, 205 p. 
Migration des espèces diadromes

Contamination de la faune aquatique à la Chlordécone et décontamination

Réseau hydrographique, cascade et intermittence du débit

Interfluve aux versants escarpés

Gradient forestier

(d'hygrophyle à xérophyle)

Agriculture diversifiée (plantation, vivrière et élevage)

Agriculture de plantation (banane ou canne à sucre)

Habitat dispersé en voie de péri-urbanisation

Espace urbain

Mangrove

Lagon

Barrière de corail

Zone pélagique

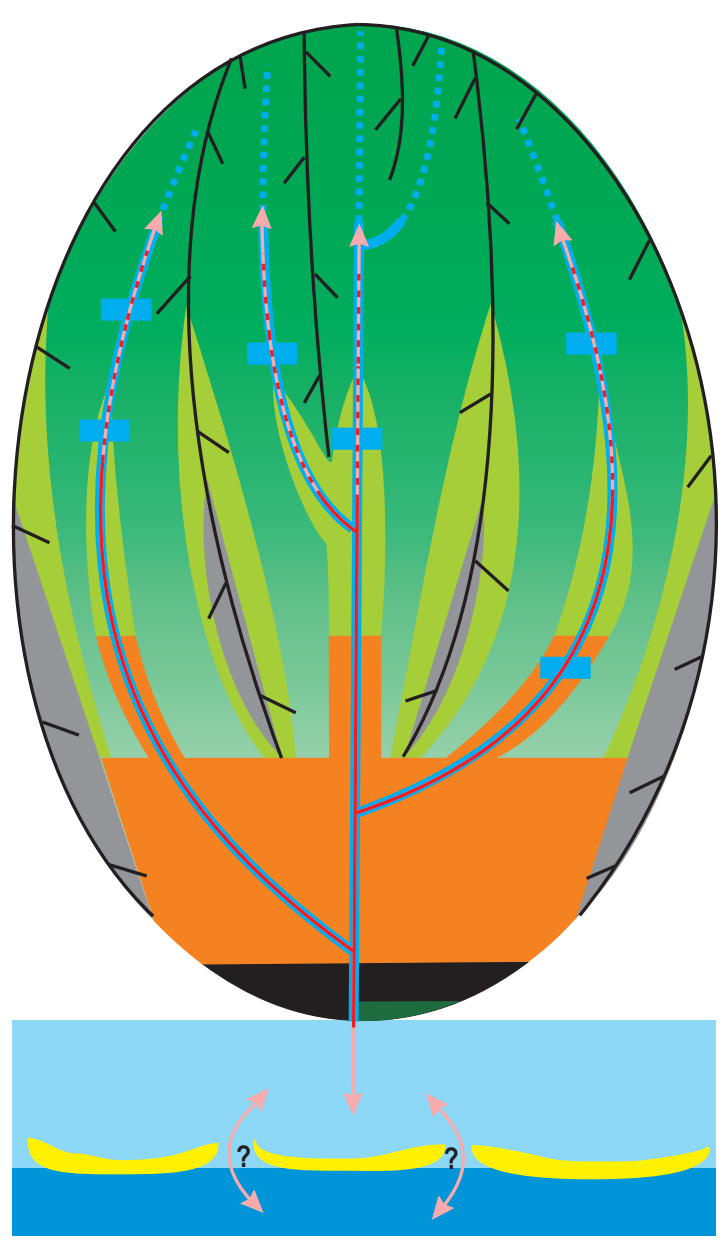

Figure 2 : L'organisation spatiale d'un bassin versant type de la Martinique et la migration des espèces aquatiques d'eau douce impactées par la Chlordécone

Spatial organisation of a typical catchment in Martinique and migration of fresh water aquatic species exposed to chlordecone 


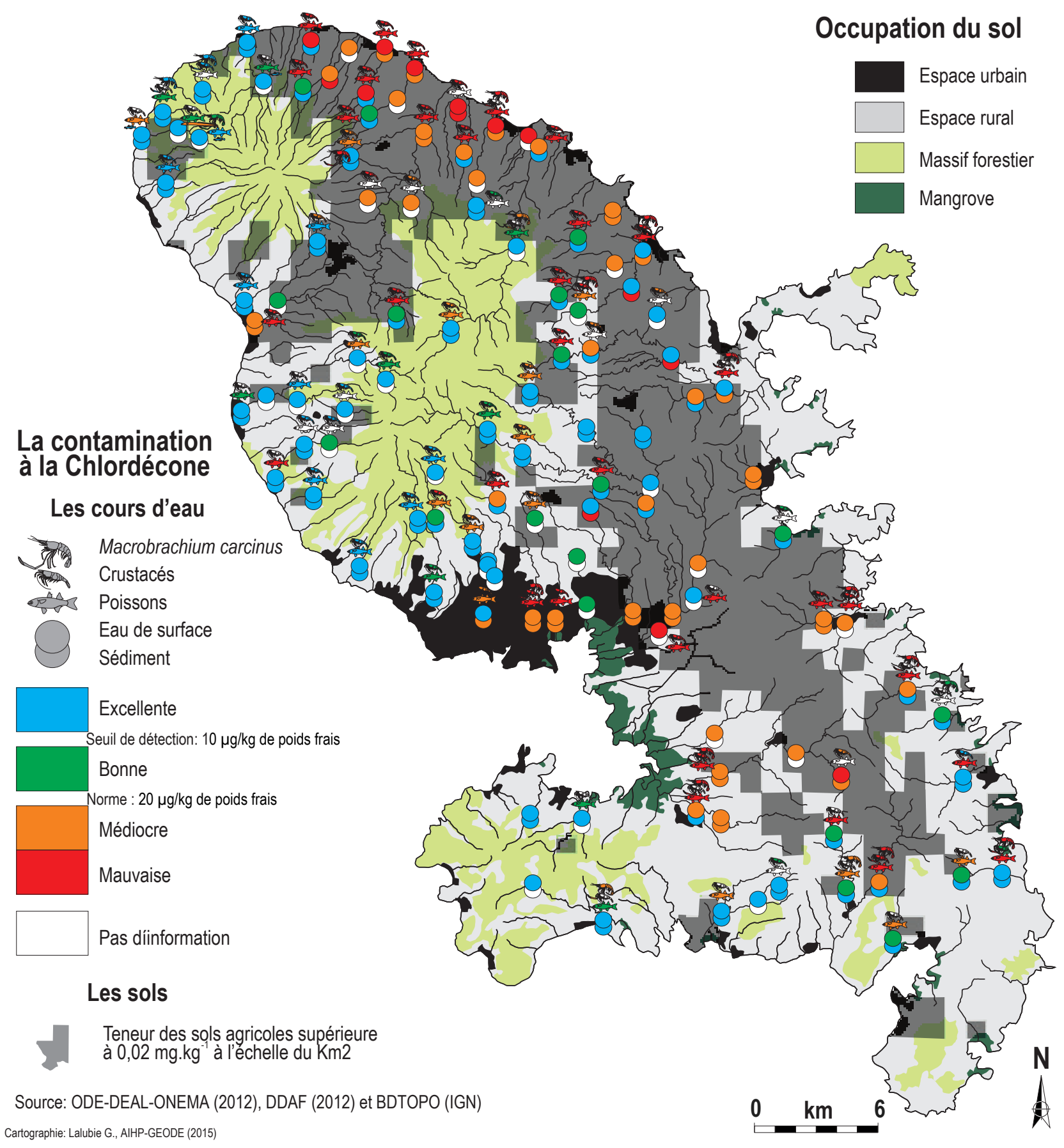

Figure 3 : La contamination par la Chlordécone du milieu terrestre à la Martinique Contamination of a terrestrial area by chlordecone in Martinique 